\title{
Controlled synthesis and structural characterization of polycrystalline GaSe
}

\author{
M. M. Abdullah · G. Bhagavannarayana • \\ M. A. Wahab
}

\begin{abstract}
A comprehensive study on the synthesis and characterization of gallium selenide (GaSe) has been reported in this paper. Synthesis of gallium selenide (GaSe) from component elements has been carried out in a specially designed and fabricated quartz ampoule, with the help of a rocking furnace. X-ray diffractometry study revealed crystalline GaSe samples belonging to hexagonal system. Raman spectral study emphasized three sharp lines at $208.89,253.95$, and $306.52 \mathrm{~cm}^{-1}$, in good agreement with X-ray diffraction results. Scanning electron microscopy revealed particles of rock like structure, sized in the range $0.4-6 \mu \mathrm{m}$.
\end{abstract}

\section{Introduction}

The III-VI layered family of semiconductors has attracted the interest of both theoretical and experimental researchers due to the presence of interactions of very different natures and for their potential applications. The most characteristic feature of this family of semiconductors is the existence of layers where bonds are covalent with a certain degree of ionic component in it and the layers are held together by weak van der Waals forces. The properties of III-VI layered compounds in ambient condition show a highly anisotropic behavior [1]. Being a member of the group III-VI, GaSe is a layered semiconductor whose $c$-axis is perpendicular to the layer planes [2]. Its layer structure consists of four close-packed monoatomic sheets in the sequence Se-Ga-Ga-Se. The strong bonding between two sheets of the same layer is supposed to be covalent with some ionic contribution. The complete fourfold layers are bound together by much weaker interlayer forces [3].

Gallium selenide ( $\mathrm{GaSe}$ ) has a number of interesting properties for electrical and non-linear optics applications. It has been reported to be used in making a number of devices like MOSFET, IR detectors, solar cells, compound semiconductor hetrostructure, etc., in crystalline form while in amorphous form, it is a potential candidate for optical memory type applications [4-6].

As a matter of fact, the literature survey revealed that the polycrystalline form of pure GaSe is least studied. In view of this, a systematic study of its synthesis and related kinetics, structural studies with the help of Powder XRD, Raman shift, and micro-structural studies using scanning electron microscopy (SEM) have been done and presented in this paper.

\section{Experimental details}

Synthesis of compound

Synthesis of GaSe has been carried out according to the solid state reaction from a conventional mixture of gallium and selenium. Bulk selenium was first properly grinded. High-purity selenium and gallium $(4 \mathrm{~N}$ and $5 \mathrm{~N}$, respectively), in stoichiometric portions, were then sealed in a transparent fused quartz ampoule with dimensions of $10 \mathrm{~mm}$ in diameter and $100 \mathrm{~mm}$ in length. The ampoule was first washed with distilled water and then rinsed with 
acetone and finally annealed at $400{ }^{\circ} \mathrm{C}$ for $4 \mathrm{~h}$. The ampoule along with conventional mixture was evacuated to $10^{-6}$ Torr using a high vacuum system. The sealed and evacuated ampoule was heated in a uniform temperature zone of a small furnace horizontally mounted on a platform, which in turn could be rocked through $\pm 30^{\circ} \mathrm{C}$ with respect to the horizontal in a cycle of $30 \mathrm{~s}$, thus ensuring proper mixing. The temperature was controlled through a programmable controller within the accuracy of $\pm 1{ }^{\circ} \mathrm{C}$ supplied by Max. Therm. The furnace was connected through a UPS of $25 \mathrm{KVA}$ to ensure a continuous power supply. In order to carry out the synthesis process, a $50 \mathrm{~h}$ program consisting of seven different runs was planned. The ampoule was first heated gradually by increasing the temperature up to $700{ }^{\circ} \mathrm{C}$ (about $15^{\circ} \mathrm{C}$ above the boiling point of selenium) for the period of $8 \mathrm{~h}$ and maintained at this temperature for $4 \mathrm{~h}$. Again the furnace temperature was allowed to increase gradually up to $1000{ }^{\circ} \mathrm{C}$ for the period of $3 \mathrm{~h}$ and maintained at this temperature for $6 \mathrm{~h}$. During this period the selenium and its possible complexes were allowed to decompose to ensure the formation of better reactants for the preparation of GaSe (melting point: $960{ }^{\circ} \mathrm{C}$ ). The furnace was then cooled gradually from 1000 to $960{ }^{\circ} \mathrm{C}$ for $2 \mathrm{~h}$ and maintained here for $4 \mathrm{~h}$. At this temperature, the furnace was rocked through $\pm 30{ }^{\circ} \mathrm{C}$ for a proper mixing of gallium and selenium. Finally the charge was brought to room temperature at a very slow rate, i.e., about half degree celsius per minute from its melting point for $23 \mathrm{~h}$. The final cooling of the material was done by switching off the furnace and left it to cool overnight. In all the above mentioned runs, the furnace was rocked from 8th hour to 12th hour then from 15th hour to 21 st hour and lastly from 23 rd hour to 27 th hour. During the intermediate periods the ampoule was left in the tilted position while heating and cooling. The synthesized material was taken out from the ampoule having natural facets shining with brown color as shown in Fig. 1. For the characterization of this material, the synthesized specimen was made powder using mortar and pestle.

\section{Characterization techniques}

The powder X-ray diffractometry (XRD) analysis of the synthesized material was carried out by using PW 1830 apparatus (PANalytical) with monochromated $\mathrm{Cu} \mathrm{K} \alpha_{1}$ radiation $(35 \mathrm{kV}, 30 \mathrm{~mA})$, scanned in step size of $0.02^{\circ}$ for the angular range $5^{\circ}-70^{\circ}$ of $2 \theta$ to know the crystal structure. The registered XRD patterns (Fig. 2) attest crystalline nature of the material. The peak width at half maximum is used to determine the crystallite size $(D)$ by using DebyScherrer formula [7]

$D=K \lambda /(\beta \cos \theta)$

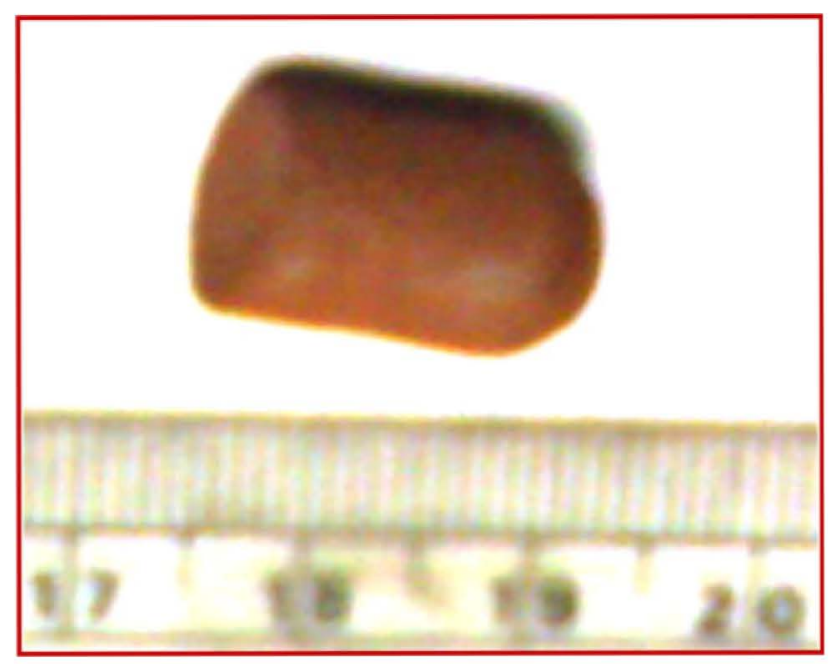

Fig. 1 As-synthesized composition of gallium selenide

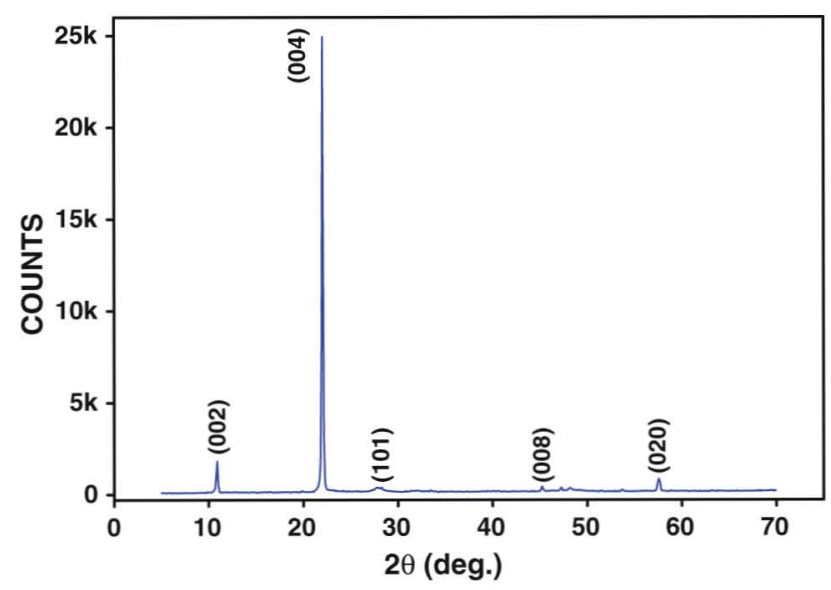

Fig. 2 Recorded pattern of polycrystalline GaSe powder in pure form

where $K=0.9$ is the Scherer constant, $\lambda=1.54056 \AA$ is the wave length of $\mathrm{X}$-ray radiation, $\beta$ is the peak full width at half maximum in radians, and $\theta$ is the Bragg diffraction angle.

For Raman measurements, polycrystalline GaSe powder was placed in a glass capillary to record the Raman spectra in the frequency range 100 to $3500 \mathrm{~cm}^{-1}$ by a PerkinElmer GX-1 Raman 2000 apparatus, working in the triple subtractive mode, equipped with a CCD detector and using a Nd-YAg laser for single excitation. On average, three scans have been recorded with a laser power $1500 \mathrm{~mW}$, measured at the laser head having a wave number resolution of $2 \mathrm{~cm}^{-1}$ at $25^{\circ} \mathrm{C}$. It is pertinent to mention that the Raman spectra could not be recorded in the range below $100 \mathrm{~cm}^{-1}$ due to experimental constraints but with no adverse effect on our results.

In the present case, the morphology and structure of the synthesized polycrystalline GaSe powder has been 
characterized with a JEOL Scanning Electron Microscope (Model No. 3300).

\section{Results and discussion}

Kinetics of synthesis

The binary combination of a group III (13) metal and a chalcogen of group VI (16) shows great diversity and many different stoichiometries [8]. This technique of combination (synthesis) basically involves the freezing of melt through a suitable temperature gradient. GaSe with desired stoichiometry is obtained as a result of the elemental combination reaction of gallium and selenium in molten state.

$\mathrm{Ga}+1 / 2 \mathrm{Se}_{2} \rightarrow \mathrm{GaSe}$

For the synthesis of this compound, the utmost attention shall be paid to ampoule geometry and symmetry, amount of element/compound, heating/cooling rate, as well as uninterrupted power supply.

It is important to note that the melting point of ampoule materials must be higher than that of the molten material and that the molten material should not be reactive with the ampoule material. Based on this principle, we have designed and fabricated the ampoule using quartz (melting point: $1650 \pm 75^{\circ} \mathrm{C}$ ) which is least reactive with $\mathrm{Ga}$ (melting point: $29^{\circ} \mathrm{C}$ ), Se (melting point: $217^{\circ} \mathrm{C}$ ) and GaSe (melting point: $960{ }^{\circ} \mathrm{C}$ ). Washing and cleaning of ampoule using distilled water and acetone help in synthesizing the impurity-free compound. Also the annealing of ampoule before synthesis increases its elastic properties, which in turn decreases the brittleness and hence reduces the probability of explosion. Selenium sublimates (around $700{ }^{\circ} \mathrm{C}$ ) below the melting point of GaSe. This results in an increased pressure exerted on the ampoule wall, which may cause even ampoule explosion. To avoid any possible explosion the wall thickness of the ampoule is optimized to $1.0 \mathrm{~mm}$ so that it can withstand the high internal pressure.

In order to obtain a homogeneous solidified alloy with at least one comparatively high vapor pressure component, the ratio of alloy-free and alloy-filled volume of ampoule should not be larger than 1:5 [9]. Under this restriction, only small quantities of samples can be synthesize because the synthesis of bulk quantities can lead to explosion particularly in the case when at least one component sublimates below the melting point. Gallium was used is in liquid form as it is in liquid state at room temperature, while selenium was properly grinded into a powder form. There are some advantages in using the source material in the powdered form viz. that the internal pressure generated during the zone melting is redistributed over the available volume in the ampoule so that the chance of explosion is reduced. However, at the same time there is also a disadvantage of this as it can decompose to a larger extent because of the free volume and temperature gradient available above the melt [10]. Removal of gaseous atmosphere inside the ampoule is extremely essential because it reduces the probability of explosion and gives an impurityfree compound.

The temperature increase during synthesis should be slow, to avoid any adiabatic change in pressure inside the ampoule, as the fast pressure increase can damage or destroy it. The sealed ampoule should be placed in the hot zone having nearly similar temperature because the temperature gradient can cause some phase change due to mass transportation inside the ampoule (therefore, rocking-type furnace is more suitable for the purpose of synthesis). Also, a large temperature gradient can damage the ampoule. Cooling of the sample to room temperature is the most important part of synthesis because there is a maximum probability for the occurrence of unwanted events like phase changes, cracks, pore/hole formation, etc. In the case of GaSe synthesis the errors induced by the above effects can be minimized up to a significant figure only by using the slow cooling method. No phase change has been observed during the actual process of synthesis. The fast cooling produces high rates of contraction for both ampoule and melt and creates high strain between melt and the ampoule wall. This strain may cause ampoule to explode or at least to crack. Therefore, the cooling should be slow to avoid any damage in the ampoule and to minimize imperfections in the compound. Each synthesis takes more than a day, therefore a provision of uninterrupted power supply should be ensured, since even a brief failure of power can cause explosion in the ampoule and introduction of imperfections in the compound.

\section{Powder XRD}

Figure 2 shows the recorded XRD patterns of pure GaSe powder. The scanning is performed at room temperature using a wavelength of $1.54056 \AA$. The obtained $d$-spacing values are in well agreement with that reported in J.C.P.D.S data file no. 37-931 attesting that as-synthesized GaSe is hexagonal of $\varepsilon$-modification [3]. The calculated lattice constants for GaSe with the help of Prozski software were found to be $a=b=3.7384 \AA$, and $c=16.0282 \AA$ and is in well agreement with the corresponding values for the specimen earlier reported (JCPDS data file no. 37-931) as $a=b=3.749 \AA$ and $c=15.907 \AA$. The most intense and sharp peaks (Fig. 2) correspond to $(0,0,2 n)$ plane which clearly depicts that the preferential orientation of crystallites are along $c$-axis. 
By using the Deby-Scherrer formula (1), we calculated the crystallite size associated with the strongest peak of (004) plane and found it to be $50.43 \mathrm{~nm}$. This is a relatively high value, which indicates the increase in the crystallinity of the synthesized GaSe.

The general aspect of the XRD patterns (Fig. 2), in particular, the presence of a single strong and sharp peak and absence of any diffraction halo, indicate that as-synthesized specimen is crystalline in nature and also show no evidence of an amorphous phase formation.

Raman study

Figure 3 shows the Raman spectra of synthesized polycrystalline gallium selenide $(\mathrm{GaSe})$ powder within the frequency range 100 to $3500 \mathrm{~cm}^{-1}$. This spectrum contains three sharp Raman lines at 208.89, 253.95, and at $306.52 \mathrm{~cm}^{-1}$ (identified as $E^{\prime \prime}, E^{\prime}$, and $A_{1}{ }^{\prime}$ modes respectively), along with a large background. Since no other Raman data for polycrystalline $\mathrm{GaSe}$ are available, our results can not be directly compared. However, the available Raman data for polycrystalline selenium (c-Se) show the presence of only two lines located at 140 and $235 \mathrm{~cm}^{-1}$ while no Raman lines have been reported to exist for $\mathrm{c}-\mathrm{Ga}$ within the frequency range 100 to $600 \mathrm{~cm}^{-1}$ [4]. The occurrence of (c-Se) Raman lines has been attributed to the (c-Se) atoms or Se-Se bonding in the structure. Further, Allakhverdiev et al. have compiled Raman data of GaSe single crystals reported by different authors together with his own results in the frequency range $0-350 \mathrm{~cm}^{-1}$ (Table 2 in [11]). From [11], it is clear that there are 4-7 Raman lines in the frequency range above $100 \mathrm{~cm}^{-1}$ and $2-5$ below $100 \mathrm{~cm}^{-1}$. They are attributed predominantly to different intralayer and (to a small extent) to interlayer bonding. In the present work, we observe three sharp and

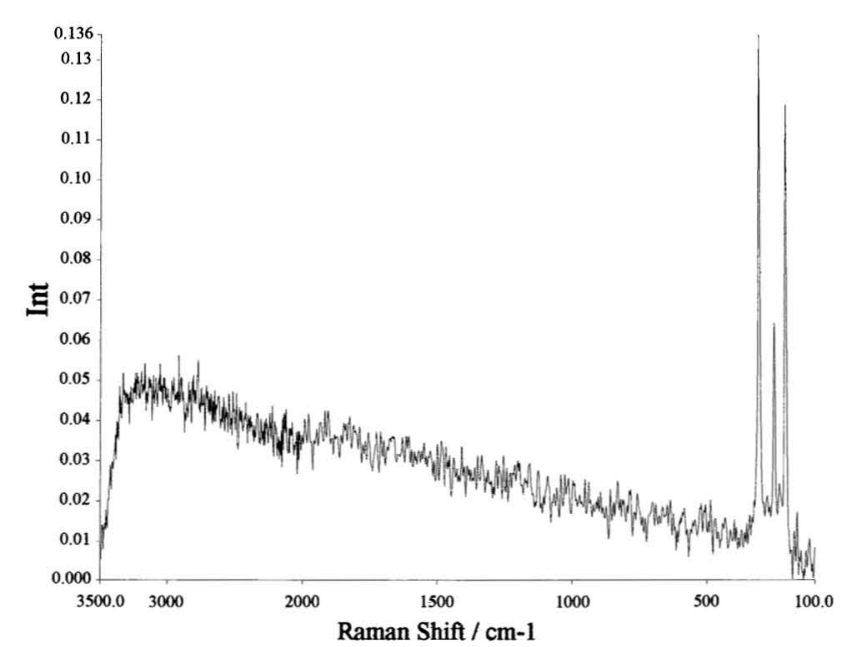

Fig. 3 Raman spectra of polycrystalline GaSe powder
Table 1 Characteristics of Raman lines observed in our experiment

\begin{tabular}{llll}
\hline $\begin{array}{l}\text { Frequency } \\
\left(\mathrm{cm}^{-1}\right)\end{array}$ & $\begin{array}{l}\text { Mode of } \\
\text { vibration }\end{array}$ & Location & $\begin{array}{l}\text { Gruneisen } \\
\text { parameters } \\
(\gamma)\end{array}$ \\
\hline 208.89 & $E^{\prime \prime}$ & Intralayer & 0.56 \\
253.95 & $E^{\prime}(\mathrm{LO})$ & Intralayer & 0.33 \\
306.52 & $A_{1}{ }^{\prime}$ & Intralayer & 0.4 \\
\hline
\end{tabular}

intense Raman lines in the range above $100 \mathrm{~cm}^{-1}$, which are identical with those reported in [11] for all GaSe single crystals. This clearly indicates that actual lines are due to different intralayer bonding in GaSe crystals. Consequently, the synthesized GaSe material in the present work is crystalline in nature. This result is in conformity with the XRD result and confirms the $\varepsilon$-modification of specimen. As far as the observation of only three sharp and intense Raman lines in the present work unlike 4-7 in single crystal cases [11], it is attributed to the fact that the powder form of synthesized material contains small-sized crystals, while data reported in [11] refer to bulk single crystalline materials. Some relevant information related to three Raman lines is provided in Table 1. The Gruneisen parameters [12] are computed by using the equation

$\gamma=-\left(\ln v / \ln V_{\mathrm{a}}\right)$

where $v$ is the frequency of atomic vibration and $V_{\mathrm{a}}$ is the atomic volume. They characterize the strength of respective intralayer bonds.

\section{Study of structure and morphology using SEM}

The scanning electron microscopy (a JEOL, model no 3300 apparatus) was used to examine the surface morphology of

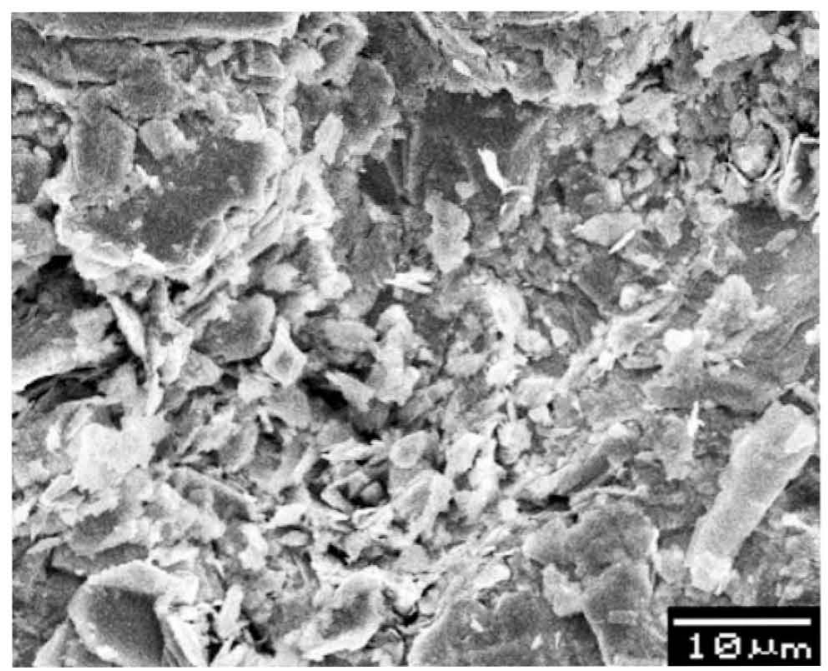

Fig. 4 SEM image of polycrystalline GaSe powder 
GaSe samples. Hardly any well-defined shapes, rather some rock like structures were observed (Fig. 4). Particle sizes are in the range of $400 \mathrm{~nm}-6 \mu \mathrm{m}$. The obtained crystallite size $(50.43 \mathrm{~nm})$ of the specimen based on XRD result is an order of magnitude smaller than the observed particle size $(400 \mathrm{~nm}-6 \mu \mathrm{m})$ of the SEM image.

\section{Conclusions}

With the help of a rocking furnace, the synthesis carried out using a specially designed and fabricated ampoule, at a low heating/cooling rate, was found to be efficient in getting a single-phase and impurity-free gallium selenide material. Further, the XRD patterns corroborated with the Raman spectra, emphasizing three strong lines located at $208.89,253.95$, and at $306.52 \mathrm{~cm}^{-1}$, explain the singlephase crystalline nature of the material. Also, the obtained crystallite size $(50.43 \mathrm{~nm})$ of the specimen based on X-ray diffraction result is an order of magnitude smaller than the observed particle size $(400 \mathrm{~nm}-6 \mu \mathrm{m})$ of the images obtained using scanning electron microscope.

\section{References}

1. Pellicer-Porres J, Segura A, Ferrer Ch, Munoz V (2002) Phys Rev B 65:174103-1

2. Seyhan A, Karabulut O, Akmoglu BG, Aslan B, Turan R (2005) Cryst Res Technol 40:893

3. Khalid Anis M, Nazar FM (1983) J Mater Sci Lett 2:471

4. Campos CEM, de Lima JC, Grandi TA, Machado KD, Pizani PS (2003) Solid State Commun 126:611

5. Singh NB, Suhre DR, Rosch W, Meyer R, Marable M, Fernelius NC, Hopkins FK, Zelmon DE, Narayanan R (1999) J Cryst Growth 198/199:588

6. Singh NB, Suhre DR, Balakrishna V, Marable M, Meyer R, Fernelius NC, Hopkins FK, Zelmon D (1998) Prog Cryst Growth Char 37:47

7. Gupta KK, Jassal M, Agrawal AK (2007) Res J Textile Apparel 11:1

8. Afzaal M, O'Brien P (2006) J Mater Chem 16:1597

9. Dietl J (Munich, DT), Jarosch J (Munich, DT) (1977) Process for preparing a homogeneous alloy. US Patent 4011074

10. Gopalakrishnan PS, Ajay C, Lakshminarasimham PS, Rangarajan K (1986) NAL Institutional Repository - Synthesis and crystal growth of IR detector materials. Ref. No. 3/2(1)/80-IV dated 27.6.1980

11. Allakhverdiev K, Baykara T, Ellialtioglu S, Hashimzade F, Huseinova D, Kawamura K, Kaya AA, Kulibekov AM, Onari S (2006) Mater Res Bull 41:751

12. Wahab MA (2005) Solid state physics, 2nd edn. Narosa Publishing House Pvt. Ltd., New Delhi, India, p 245 